\title{
PERTURBATION APPROACH TO THE FERMI GAS MODEL OF HEAVY NUCLEI
}

\author{
by N. M. HUGENHOLTZ
}

Instituut voor theoretische fysica der Rijksuniversiteit, Utrecht, Nederland

\section{Synopsis}

The general perturbation method presented in an earlier paper ${ }^{1}$ ) is applied to a study of some aspects of the Fermi gas model of nuclear matter. The two extreme cases of very low and very high particle density are investigated. It is shown that the theory recently developed by Brueckner ${ }^{2}$ ) can be considered as an improved low density approximation. The validity of some other approximations made in the Brueckner theory, involving the energy denominators in intermediate states are briefly discussed. The study of the case of high density reveals the interesting fact that, contrary to indications presented by Swiatecki ${ }^{3}$ ) and Bethe ${ }^{4}$ ), the convergence of the perturbation expansion gets worse with increasing density, while in that case the Brueckner approximation becomes extremely poor.

It is furthermore shown that a slow nucleon, travelling through nuclear matter, can be considered as being in a metastable state and an exact expression for the inverse life-time of such a state is given; this quantity must be identified with the imaginary part of the potential in the optical model.

1. Introduction. The Fermi gas model of heavy nuclei, which forms the subject of the present paper, is meant to give a description of properties which are independent of the size or the detailed structure of the individual nuclei. It is to be expected that some properties of nuclear matter, in the interior of large nuclei, such as the binding energy per nucleon or the shift of the energy of an additional nucleon penetrating into the interior of a nucleus, can be studied by considering a large box filled with interacting nucleons, with a particle density equal to the density of actual nuclei. This model might be called the Fermi gas model of nuclear matter.

In the past there have been several attempts to make calculations of the binding energy of heavy nuclei on the basis of the Fermi gas model, in particular by Euler and by $\cdot \mathrm{Huby}^{5}$ ). The interaction between the nucleons was treated as a perturbation and the binding energy per nucleon was calculated to second order in the interaction. The results of such calculations were not very encouraging and seemed to confirm the general believe that, due to the strong correlations between the nucleons, perturbation theory is inadequate for the treatment of nuclei.

Recently Swiatecki ${ }^{3}$ ) re-examined the calculation of Euler. He studied the ratio $\Delta E_{0}^{(2)} / \Delta E_{0}^{(1)}$ of the second and first order terms of the binding 
energy $\Delta E_{0}$ as a function of the particle density of the nucleon gas. He showed that this ratio decreases rapidly with the density. Hence, in the limit of large density, the first order term becomes predominant. This behaviour raised the hope that, in the case of large densities, the perturbation expansion would converge well. This expectation however is not confirmed, as will be shown in the present paper.

New interest has been aroused in the Fermi gas model by Brueckner e.a. $\left.{ }^{2}\right)$ who proposed a new approach to the many particle problem. The method of Brueckner can be considered as an improved Hartree method. The interaction between two nucleons is treated exactly, whereas the influence of all other particles is taken into account by means of a selfconsistent Hartree potential. The method aims at giving the theoretical foundation of the shell model. Although attempts have been made to adapt the method to the treatment of finite nuclei, the method of Brueckner is in essence an approximation method for the imperfect Fermi gas. There has been much discussion on the limits of validity of the Brueckner approximation, but this difficult question is far from settled.

A detailed study of the Fermi gas using perturbation theory was always hampered by the fact that the conventional perturbation theory is very inadequate for the treatment of many particle systems. In particular the occurrence of terms containing arbitrarily high powers of the number of particles in the perturbation expansion was a well known difficulty. In a recent paper ${ }^{1}$ ) (to be referred to as I) a perturbation formalism was described which is particularly suited for the investigation of systems with many degrees of freedom. In the present paper several of the results of I, where the theory of the Fermi gas was already considered as an example, will be applied to some aspects of the nuclear structure problem.

We base our discussion on a consideration of the two limiting cases of very small and very large densities. In section 2, the case of small density (the average distance between the particles is then large compared to the range of the forces) will be studied. It is shown how, starting from this extreme case, the Brueckner approximation presents itself in a natural way as an approximation for not too high densities. In section 3 we study the opposite case where the particle density is high. The leading term of the $n$th order $(n \geqslant 2)$ contribution to $\Delta E_{0}$ is shown to be proportional to $k_{F}^{n+1}$, where the Fermi momentum $k_{F}$ is used as a measure for the particle density. This shows that, in the case of high particle density, the perturbation expansion gets worse with increasing density. In the same section the $k_{F^{-}}$ dependence of the different terms of the Brueckner approximate expansion are calculated asymptotically for large $k_{F}$, yielding for the $n$th order term the factor $k_{F}^{5-n}$. Consequently the Brueckner approximation is very poor and misleading at high densities because it leaves out completely the leading effects in this density region. 
In section 4 the motion of an additional nucleon with momentum $|k|>k_{F}$ through nuclear matter is investigated. This is of interest in connection with the optical model for the scattering of nucleons on heavy nuclei. Under some very reasonable assumptions, a state with an additional particle with momentum $|k|>k_{F}$ is shown to be metastable. The expression derived in I for the inverse life-time of such a state, a quantity which corresponds to the complex part of the potential in the optical model, leads in lowest order of approximation to a formula derived by Brueckner, Eden and Francis ${ }^{6}$.

2. The case of low particle density. In I the energy shift $\Delta E_{0}$ of the ground state of the Fermi gas, caused by the interparticle interaction $V$, was found to be (see I 9.25)

$$
\Delta E_{0}=\left\langle\varphi_{0}\left|\left[V-V\left(H_{0}-\varepsilon_{0}\right)^{-1} V+\ldots\right]_{C}\right| \varphi_{0}\right\rangle .
$$

For the meaning of the symbols one is referred to I. By this formula the binding energy $-\Delta E_{0}$ is expressed in contributions of connected ground state diagrams only. Consequently, for large volume $\Omega, \Delta E_{0}$ is asymptotically proportional to $\Omega$.

If we take spinless particles with central two-body forces (the extension to the case of particles with spin will be given when necessary) the first term of (2.1) gives

$$
\Delta E_{0}^{(1)}=\frac{1}{2} \Omega(2 \pi)^{-6} \int_{0}^{k_{F}} \mathrm{~d}^{3} m_{1} \int_{0}^{k_{F}} \mathrm{~d}^{3} m_{2}\left(v(0)-v\left(m_{1}-m_{2}\right)\right) .
$$

The integration in (2.2) is extended over all momenta $m_{1}$ and $m_{2}$ inside the Fermi sphere of radius $k_{F}$ (As in I we use the convention that the symbols $k_{i}\left(m_{i}\right)$ denote momenta outside (inside) the Fermi sphere.). If $k_{F}$ is very small, corresponding to the case of low density, the integrand can be expanded in powers of $\left|m_{1}-m_{2}\right|$. We get in lowest order

$$
\Delta E_{0}^{(1)}=-\frac{1}{2} \Omega(2 \pi)^{-6} \int_{0}^{k_{F}} \mathrm{~d}^{3} m_{1} \int_{0}^{k_{F}} \mathrm{~d}^{3} m_{2}\left|m_{1}-m_{2}\right| v^{\prime}(0) .
$$

This expression is proportional to $k_{F}^{7}$, six factors $k_{F}$ arising from the 6-fold integration and one from the integrand. This last factor would be absent if we had taken Serber forces between particles with spin $\frac{1}{2}$. The major change in the latter case would be a plus sign in the interaction $V$ instead of a minus sign, and the integrand in (2.2) would not vanish for small $\left|m_{1}-m_{2}\right|$.

It is now very easy to see which higher order diagrams give the largest contribution to $\Delta E_{0}$ for small $k_{F}$. They are the diagrams with the smallest possible number of hole lines. In each order in $V$ there is always exactly one diagram with only two such lines. They are of the type as shown in figure $1 a$. It is easily established that the contributions of these diagrams (except the lowest order one) are proportional to $k_{F}^{8}$, two factors $k_{F}$ arising from the integrand. In the more realistic case of Serber forces the diagrams 
considered would give a contribution of order $k_{F}^{6}$. All other diagrams give contributions containing higher powers of $k_{F}$ and can be neglected in the case of very low density.

The leading diagrams for low density are seen to correspond to iterated collisions between two particles. This result has nothing surprising. Indeed, in the kinetic theory of dilute gases it is well known that all but binary collisions can be neglected.

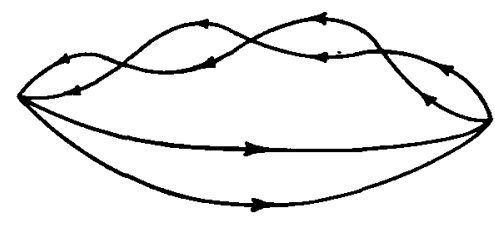

a

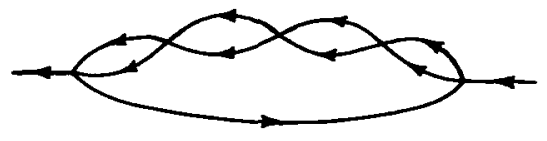

b

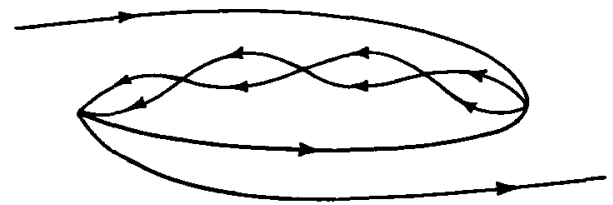

c

Fig. 1. The diagrams giving the main contribution in the case of extreme low"density. The diagrams $a, b$ and $c$ respectively correspond to the ground state, a state with an additional particle and a state with a hole.

For states with the Fermi sea and one additional particle of momentum $k$, or with one hole of momentum $m$ in the Fermi sea, one has to study the functions $\bar{G}_{k}\left(\varepsilon_{0}+z\right)$ or $\bar{G}_{m}\left(\varepsilon_{0}+z\right)$, i.e., the function $\bar{G}_{\alpha}\left(\varepsilon_{0}+z\right)$ defined in (I 10.2) taken for a state $|\alpha\rangle$ equal to $|k ;\rangle$ or $|; m\rangle$. The diagrams contributing to these functions have one external line at both ends. Exactly as before it is seen that the diagrams $b$ and $c$ of fig. 1 give, in the case of low density, the main contributions to $\bar{G}_{k}\left(\varepsilon_{0}+z\right)$ and $\bar{G}_{m}\left(\varepsilon_{0}+z\right)$ respectively. They all have one internal hole line.

In the approximation where the contributions of all other diagrams are neglected, the energy denominators $\left(\varepsilon_{\gamma}-\varepsilon_{0}\right)^{-1}$ and $\left(\varepsilon_{\gamma}-\varepsilon_{0}-z\right)^{-1}$ for the intermediate states $|\gamma\rangle$ occurring in $\Delta E_{0}, \bar{G}_{k}\left(\varepsilon_{0}+z\right)$ and $\bar{G}_{m}\left(\varepsilon_{0}+z\right)$ contain the unperturbed energies $\varepsilon_{\gamma}-\varepsilon_{0}$. If one wants to improve the approximation and incorporate in the intermediate states the energy shift of the additional particles caused by the presence of all other particles in the gas, the following extension of the low density approximation presents itself in a natural way.

Instead of equation (I 9.25) for the energy shift of the ground state, we take (I 9.26) as our starting point:

$$
\Delta E_{0}=\left\langle\varphi_{0}\left|\left[V-V \bar{D}\left(\varepsilon_{0}\right) V+\ldots \ldots\right]_{\ell}\right| \varphi_{0}\right\rangle .
$$


Here the matrix element involves the contributions of all irreducible connected ground state diagrams. The factors $\bar{D}_{\gamma}\left(\varepsilon_{0}\right)$, which replace the factors $\left(\varepsilon_{y}-\varepsilon_{0}\right)^{-1}$ in the intermediate states of $(2.1)$, are obtained from (I 10.3)

$$
\bar{D}_{\gamma}\left(\varepsilon_{0}+z\right)=\left[\varepsilon_{\gamma}-\varepsilon_{0}-z-\bar{G}_{\gamma}\left(\varepsilon_{0}+z\right)\right]^{-1}
$$

where

$$
\delta\left(\gamma-\gamma^{\prime}\right) \bar{G}_{\gamma}\left(\varepsilon_{0}+z\right)=\left\langle\gamma^{\prime}\left[-V+V \bar{D}\left(\varepsilon_{0}+z\right) V-\ldots\right]_{i a L} \mid \gamma\right\rangle .
$$

In the latter equation only irreducible diagrams without ground state components are taken into account. The announced extension of the low density approximation is obtained if the irreducible diagrams occurring in (2.3) are restricted to the type $a$ of fig. 1, while the irreducible diagrams in (2.5) are restricted to diagonal diagrams the components of which are of the type $b$ and $c$. This approximation represents a considerable improvement above the original low density approximation.

As will be shown in section 4 the functions $\bar{D}_{\gamma}\left(\varepsilon_{0}+z\right)$ for $|\gamma\rangle \neq\left|\varphi_{0}\right\rangle$ have no pole. However, for small excitation energies, one has in good approximation

$$
\bar{D}_{\gamma}\left(\varepsilon_{0}+z\right)=\bar{N}_{\gamma}\left(\bar{E}_{\gamma}-z\right)^{-1}+\bar{\psi}_{\gamma}\left(\varepsilon_{0}+z\right) .
$$

with $\bar{\psi}_{\gamma}$ small for $z=\bar{E}_{\gamma} . \bar{N}_{\gamma}$ is defined in (I 10.9). $\bar{E}_{\gamma}$ is approximately the perturbed excitation energy of the state $|\gamma\rangle$; as shown in I section 10, it is the sum of the perturbed energies of the individual particles and holes of $|\gamma\rangle$. The equations become formally much simpler if in all intermediate states $|\gamma\rangle$ of (2.3) and (2.5) we put approximately

$$
\bar{D}_{\gamma}\left(\varepsilon_{0}+z\right)=\left(\bar{E}_{\gamma}-z\right)^{-1} \text {. }
$$

If this is done (we come back later on the error involved in this step), the improved low density approximation, described above, becomes equivalent to the Brueckner approximation. To show this equivalence we calculate $\Delta E_{0}$ and the functions $\bar{G}_{k}\left(\varepsilon_{0}+z\right), \bar{G}_{m}\left(\varepsilon_{0}+z\right)$ from (2.3), (2.5) and (2.6), thereby limiting ourselves to diagrams of the type of figure 1 . We find

$$
\begin{aligned}
& \Delta E_{0}=\frac{1}{2} \int_{m_{1} m_{2}} v\left(m_{1} m_{2} m_{2} m_{1}\right)+\frac{1}{4} \int_{m_{1} m_{2} k_{1} k_{2}} \frac{v\left(m_{1} m_{2} k_{1} k_{2}\right) v\left(k_{1} k_{2} m_{2} m_{1}\right)}{\bar{E}_{k_{1}}+\overline{\bar{E}}_{k_{2}}+\overline{\bar{E}}_{m_{1}}+\overline{\bar{E}}_{m_{2}}}+\ldots \\
& \delta\left(k^{\prime}-k\right) \bar{G}_{k}\left(\varepsilon_{0}+z\right)=-\int_{m_{1}} v\left(k^{\prime} m_{1} m_{1} k\right)-\frac{1}{2} \int_{m_{1} k_{1} k_{2}} \frac{v\left(k^{\prime} m_{1} k_{1} k_{2}\right) v\left(k_{1} k_{2} m_{1} k\right)}{\overline{\bar{E}}_{k_{1}}+\bar{E}_{k_{2}}+\overline{\bar{E}}_{m_{1}}-z}+\ldots \\
& \delta\left(m^{\prime}-m\right) \bar{G}_{m}\left(\varepsilon_{0}+z\right)=\int_{m_{1}} v\left(m m_{1} m_{1} m^{\prime}\right)+\frac{1}{2} \int_{m_{1} k_{1} k_{2}} \frac{v\left(m m_{1} k_{1} k_{2}\right) v\left(k_{1} k_{2} m_{1} m^{\prime}\right)}{\overline{\bar{E}}_{k_{1}}+\overline{\bar{E}}_{k_{2}}+\bar{E}_{m_{1}}+2 \bar{E}_{m}-z}+. .
\end{aligned}
$$

Let us define a quantity $G\left(l_{1} l_{2} l_{3} l_{4}\right)$, depending on the 4 momenta $l_{1} l_{2} l_{3} l_{4}$, by the integral equation

$$
G\left(l_{1} l_{2} l_{3} l_{4}\right)=v\left(l_{1} l_{2} l_{3} l_{4}\right)+\frac{1}{2} \int \mathrm{d}^{3} k_{1} \mathrm{~d}^{3} k_{2} \frac{v\left(l_{1} l_{2} k_{1} k_{2}\right) G\left(k_{1} k_{2} l_{3} l_{4}\right)}{\bar{E}_{k_{1}}+\bar{E}_{k_{2}}+\bar{E}_{m_{1}}+\bar{E}_{n_{2}}},
$$


where it should be remembered that the energy $\bar{E}_{m}$ of a hole is negative. $G\left(l_{1} l_{2} l_{3} l_{4}\right)$ is the modified scattering matrix of Brueckner. The factor $\frac{1}{2}$ in the second term on the right-hand side takes care of the fact thateach intermediate state is counted twice. The above equations now reduce to the simple form

$$
\begin{gathered}
\Delta E_{0}=\frac{1}{2} \int_{m_{1} m_{2}} G\left(m_{1} m_{2} m_{2} m_{1}\right), \\
\delta\left(k^{\prime}-k\right) \bar{G}_{k}\left(\bar{E}_{k}\right)=-\int_{m_{1}} G\left(k^{\prime} m_{1} m_{1} k\right), \\
\delta\left(m-m^{\prime}\right) \bar{G}_{m}\left(\bar{E}_{m}\right)=\int_{m_{1}} G\left(m m_{1} m_{1} m^{\prime}\right) .
\end{gathered}
$$

As shown in I, section $10,-\bar{G}_{k}\left(\bar{E}_{k}\right)$ and $-\bar{G}_{m}\left(\bar{E}_{m}\right)$ represent the energy shifts of the particle and hole respectively. If we define the diagonal matrix $\left\langle l^{\prime}|W| l\right\rangle=\delta\left(l^{\prime}-l\right) W(l)$ by

$$
\begin{aligned}
& W(k)=-\bar{G}_{k}\left(\bar{E}_{k}\right) \text { for }|k|>k_{F} \\
& W(m)=\bar{G}_{m}\left(\bar{E}_{m}\right) \text { for }|m|<k_{F},
\end{aligned}
$$

equation (2.8) and (2.9) can be written

$$
\delta\left(l^{\prime}-l\right) W(l)=\int \mathrm{d}^{3} m G\left(l^{\prime} m m l\right) .
$$

The total perturbed energy of a single particle, with momentum $l$ either outside or inside the Fermi sea (in the latter case this energy is defined as minus the energy of the hole), is thus given by

$$
|l|^{2} / 2 M+W(l)
$$

Finally, by (2.7) and (2.9), the energy shift $\Delta E_{0}$ of the ground state of the system is

$$
\Delta E_{0}=\frac{1}{2} \Sigma_{m} W(m)=\frac{1}{2} \Omega(2 \pi)^{-3} \int_{0}^{k_{F}} \mathrm{~d}^{3} m W(m) .
$$

In these equations we used the definitions for the symbols $\delta\left(m^{\prime}-m\right)$ and $\int_{m}$ introduced in I section 2. The formulae (2.10), (2.11), and (2.12) correspond with the formulae (9.14), (9.1), and (9.3) of Bethe's presentation of the Brueckner theory ${ }^{4}$ ).

A remark must still be made on the approximation (2.6), i.e. the replacement of $\bar{D}_{\gamma}\left(\varepsilon_{0}+z\right)$ by $\left(\bar{E}_{\gamma}-z\right)^{-1}$. In I section 10 the following formula was derived for $\bar{N}_{\gamma}$ :

$$
\bar{N}_{\gamma}^{-1}=1+\bar{G}_{\gamma}^{\prime}\left(\varepsilon_{0}+\bar{E}_{\gamma}\right) \text {. }
$$

Hence, the deviation of $\bar{N}_{\gamma}$ from unity is given by $\bar{G}_{\gamma}^{\prime}\left(\varepsilon_{0}+\bar{E}_{\gamma}\right)$, a quantity which, for small density, has exactly the same $k_{F}$-dependence as the energy shift $\bar{G}_{\gamma}\left(\varepsilon_{0}+\bar{E}_{\gamma}\right)$. If the latter is taken into account as a correction to the energy in the intermediate state $|\gamma\rangle$ there is no reason to neglect the former. The same holds true for the function $\bar{\psi}_{\gamma}\left(\varepsilon_{0}+z\right)$. Although it might well happen that by accidental cancellations the replacement (2.6) would be justified, there is no indication to this effect and one should consequently not be surprised to find considerable errors brought in by this approximation. 
3. The case of high density. We investigate in this section how the various terms in the simple perturbation expansion (2.1) of $\Delta E_{0}$ behave for high density, i.e., how they depend on the Fermi momentum $k_{F}$ in the case of large $k_{F}$. We assume the interparticle potential not to be too singular. For high densities the Fermi sphere has a large radius and the transitions take place only near its surface. Consequently there is a complete symmetry between holes and particles in all terms of (2.1), except the first. The contribution of a diagram (of order $n \geqslant 2$ ) is not changed if in that diagram all particle lines are replaced by hole lines, and conversely. The first order term of (2.1) forms an exception because in the interaction $V$ the $\xi$-operators precede the $\xi^{*}$-operators (in the order from right to left).

This term was already calculated in section 2 and is given by (2.2). In the present case of large $k_{F}$ the leading term of (2.2) is clearly the first. In the second term, in view of the factor $v\left(m_{1}-m_{2}\right), m_{1}$ and $m_{2}$ are bound to be close together (relatively to $k_{F}$ ), which gives a strong restriction on the domain of integration. Neglecting the second term in (2.2) the energy shift per particle is found to be

$$
\Delta E_{0}^{(1)} / N=v(0) k_{F}^{3} / 12 \pi^{2},
$$

where we used formula (I 2.9) for the particle number $N$.

There is only one second order diagram contributing to $\Delta E_{0}$ in (2.1), the contribution being given by

$$
\begin{aligned}
\Delta E_{0}^{(2)} & =-M \Omega(2 \pi)^{-9} \int \mathrm{d}^{3} k_{1} \mathrm{~d}^{3} k_{2} \mathrm{~d}^{3} m_{1} \mathrm{~d}^{3} m_{2} . \\
& . \frac{v\left(k_{1}-m_{1}\right)^{2}-v\left(k_{1}-m_{1}\right) v\left(k_{1}-m_{2}\right)}{k_{1}^{2}+k_{2}^{2}-m_{1}^{2}-m_{2}^{2}} \delta\left(k_{1}+k_{2}-m_{1}-m_{2}\right),
\end{aligned}
$$

as is easily found by the method described in I, section 3 . The integration is extended over momenta $k_{1}$ and $k_{2}$ outside the Fermi sphere and over $m_{1}$ and $m_{2}$ inside it. Let us compare the first and second term in (3.2). In the first term, through the factor $v\left(k_{1}-m_{1}\right)^{2}$, the momenta $k_{1}$ and $m_{1}$ must be close together, and, through the factor $\delta\left(k_{1}+k_{2}-m_{1}-m_{2}\right)$, also $k_{2}$ and $m_{2}$. Hence, remembering that $\left|k_{1}\right|,\left|k_{2}\right| \geqslant k_{F}$ and $\left|m_{1}\right|,\left|m_{2}\right| \leqslant k_{F}$, for the integrand to have an appreciable value, the four momenta must be close to the surface of the Fermi sphere, where two of them (e.g., $m_{1}$ and $m_{2}$ ) may be chosen arbitrarily. The second term in (3.2) contains the factor $v\left(k_{1}-m_{1}\right) v\left(k_{1}-m_{2}\right)$. There all four momenta must remain in the neighbourhood of each other at the surface of the Fermi sphere, where only one of them can be chosen arbitrarily. Clearly, this term is small compared to the first.

We shall now calculate the main term. Putting $k_{1}=m_{1}+q$ and $k_{2}=m_{2}-q$, and remembering that the values of $q$ that contribute to 
(3.2) are small compared to $k_{F}$ one can write

$$
\Delta E_{0}^{(2)}=-M \Omega(2 \pi)^{-9} \int \mathrm{d}^{3} q \mathrm{~d}^{3} m_{1} \mathrm{~d}^{3} m_{2} \frac{v(q)^{2}}{2 k_{F} q\left(\mu_{1}-\mu_{2}\right)} .
$$

In the denominator, powers of $q$ higher than the first are neglected. The variables $\mu_{1}$ and $\mu_{2}$ are the cosines of the angles of $m_{1}$ and $m_{2}$ with the vector $q$, which is chosen in the $z$-direction. The integration over $m_{1}$ and $m_{2}$ is restricted by the requirement that the momenta $k_{1}=m_{1}+q$ and $k_{2}=m_{2}-q$ lie outside the Fermi sphere. Always neglecting higher powers of $q / k_{F}$ one finds after some simple manipulations

$$
\Delta E_{0}^{(2)} / N=-\frac{3}{2} M(2 \pi)^{-4} \int_{0}^{\infty} \mathrm{d} q q^{3} v(q)^{2} \int_{0}^{1} \mathrm{~d} \mu_{1} \int_{0}^{1} \mathrm{~d} \mu_{2} \mu_{1} \mu_{2}\left(\mu_{1}+\mu_{2}\right)^{-1} .
$$

This term is independent of $k_{F}$ (in the limit of large $k_{F}$ ) whereas (3.1) is proportional to $k_{F}^{3}$. In the case of high density the first order term (3.1) is much larger than the term of second order, in accordance with the conclusion of Swiatecki mentioned in the introduction. However, as we shall see now, some of the terms of higher order in the perturbation contain increasing powers of $k_{F}$.

We consider an arbitrary connected ground state diagram of order $n$, and investigate how the contribution of this diagram depends on $k_{F}$, in the limit of large $k_{F}$. The momentum transfer $q$ in each interaction $V$ is small compared to $k_{F}$. In order to find the number of factors $k_{F}$, we may put simply $q=0$. Then each interaction $V$ (corresponding to a vertex in the diagram) gives rise to two relations, each of them relating the momentum of an incoming line to the momentum of an outgoing line in that vertex. These relations restrict the number of independent variables in the integration, thereby reducing the number of factors $k_{F}$ in the result. Such relations can be of three types: $m_{i}=m_{\mathrm{j}}, k_{i}=k_{\mathrm{j}}$ and $k_{i}=m_{\mathrm{j}}$. Each relation of the first two types reduces the number of independent integration variables by three. A relation of the latter type, however, gives one additional constraint because both $k_{t}$ and $m_{j}$ must be near the surface of the Fermi sphere. In this case the number of independent integration variables is decreased by four. One should notice that the relations corresponding to different points of the diagram can be identical. This; in fact, sometimes reduces the total number of restrictions on the integration variables considerably. Finally, each energy denominator contains a factor $k_{F}$ (as in (3.3)), which gives an extra factor $k_{F}^{1-n}$ in the whole contribution.

Counting in this way the number of factors $k_{F}$ of the leading term of each diagram, one obtains the following result. For arbitrary order $n \geqslant 2$ the diagrams of the type shown in figure 2 give, for large density, the main contribution to $\Delta E_{0}$. As will be shown now, this contribution is of order $k_{F}^{n+1}$. For the relations between the momenta in each vertex we take the following 
choice:

$$
k_{i}=m_{i},(i=1,2, \ldots, n) .
$$

The momenta $m_{i}$ can be integrated independently over the surface of the Fermi sphere, which leads to a $2 n$-fold integration, i.e., a factor $k_{F}^{2 n}$. Together with the factor $k_{F}^{1-n}$, arising from the energy denominators, we obtain the total factor $k_{F}^{n+1}$. For the energy shift per particle one can write

$$
\Delta E_{0}^{(n)} / N \sim k_{F}^{n-2},
$$

a result in agreement with our earlier conclusion concerning $\Delta E_{0}^{(2)}$.

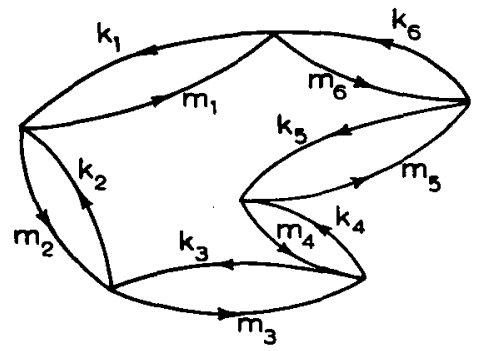

a

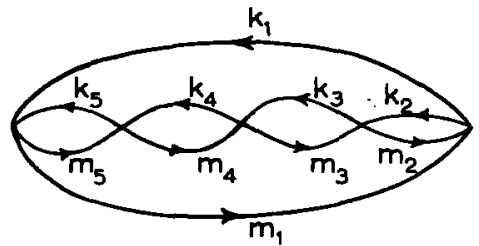

b

Fig. 2. This figure shows the type of diagrams giving the main contribution to $\Delta E_{0}$ in the case of large density. Diagram $a$ is an arbitrary example of this type. The contribution of the simple diagram $b$ is calculated in the text.

The diagram shown in figure $2 b$ is a very simple example of the type considered. A straightforward extension of the calculation of (3.4) gives the following result:

$$
\begin{aligned}
\Delta E^{(n)} / N=(-1)^{n-1} 3(2 \pi)^{-2 n} M^{n-1} k_{F}^{n-2} J \mathrm{~d} q q^{3} v(q)^{n} \\
\\
\quad \int_{0}^{1} \mathrm{~d} \mu_{1} \ldots \int_{0}^{1} \mathrm{~d} \mu_{n} \mu_{1} \ldots \mu_{n}\left(\mu_{1}+\mu_{2}\right)^{-1} \ldots\left(\mu_{1}+\mu_{n}\right)^{-1},
\end{aligned}
$$

which is valid for $n \geqslant 3$. For $n=2$ a factor $\frac{1}{2}$ must be added to account for the two pairs of equivalent lines one has in that case (see I section 3). Apart from this factor (3.6) reduces to (3.4) for $n=2$. We see further that (3.6) is in agreement with (3.5).

Before commenting on these results we shall calculate the $k_{F}$-dependence of the contributions of the Brueckner diagrams of fig. $1 a$. The $n$ th-order diagram of this type contains, in the limit of large density, the factor $k_{F}^{5-n}$. For the energy shift per particle, when calculated from these diagrams only, we find for $n \geqslant 2$

$$
\Delta E_{0}^{(n)} / N \sim k_{F}^{2-n} \text { (Brueckner approximation), }
$$

a contribution which, for large $k_{F}$, decreases rapidly with $n$. It is negligible compared to the main contribution of the same order for high density. It is even very easy to see that the Brueckner diagrams, together with the dia- 
grams obtained from them by interchanging holes and particles, give the smallest contributions in this case.

The conclusion of the above considerations is shown in the following table, where for each order $n$ in the perturbation we give the highest power of $k_{F}$ occurring in the total energy per particle $E_{0} / N$ and also the power which would be found from the Brueckner diagram of the same order.

\begin{tabular}{|l|l|l|l|l|r|r|r|}
\hline Order $n=$ & 0 & 1 & 2 & 3 & 4 & 5 & 6 \\
\hline Main term & 2 & 3 & 0 & 1 & 2 & 3 & 4 \\
Brueckner term & 2 & 3 & 0 & -1 & -2 & -3 & -4 \\
\hline
\end{tabular}

One sees that the terms in the perturbation expansion contain higher and higher powers of $k_{F}$, so that the convergence of the expansion gets worse with increasing density. It is furthermore seen that comparison of the first order with the second order term in the expansion is highly misleading as a test for overall convergence.

In order to study the validity of the Brueckner approximation Bethe ${ }^{4}$ ) calculated the expression (3.6) in 3rd order in the perturbation. His result agrees with ours, apart from some constant factors arising from the fact that he uses Serber forces. It depends linearly on $k_{F}$ for large density. Thus it is indeed small compared to the first order term of the Brueckner approximation, but it is large compared to the Brueckner term of the same order. Our general results show clearly that the Brueckner approximation necessarily is very poor in the case of large densities.

The density of nuclear matter falls in between the two extreme regions considered in the previous and present section. It is certainly not excluded that the Brueckner method, which is in essence an improved approximation method for low densities, is still applicable to actual nuclear matter, at least for qualitative purposes, but one should realize that this important issue cannot be decided on the basis of the numerical calculations, published so far by the various authors working in this field. It nevertheless seems clear that the Brueckner method should not be expected to achieve any degree of quantitative accuracy.

4. On the metastable character of the excited states. It was already stated in I that, in the theory of an imperfect Fermi gas, none of the perturbed states, distinct from the ground state, are exactly stationary. In this section we shall show how this conclusion can be reached. We shall base our discussion on the considerations in $I$, in particular sections 10 and 14 .

As shown there, to each unperturbed state $|\alpha\rangle \neq\left|\varphi_{0}\right\rangle$ there corresponds a quantity $\bar{E}_{\alpha}$, which is defined as the root of equation (I 10.6). This quantity $\bar{E}_{\alpha}$ is the energy of a stationary state of the perturbed system, provided the function $\bar{G}_{\alpha}\left(\varepsilon_{0}+z\right)$ is regular at the point $z=\bar{E}_{\alpha}$. If, on the other hand, 
$\bar{G}_{\alpha}\left(\varepsilon_{0}+z\right)$ is discontinuous for $z$ crossing the real axis at $\bar{E}_{\alpha}$, this energy has in general no simple physical meaning. If, however, the discontinuity of $\bar{G}_{\alpha}\left(\varepsilon_{0}+z\right)$ at $z=\bar{E}_{\alpha}$ is small, $\bar{E}_{\alpha}$ is the (approximate) energy of a metastable state. The discontinuous change of $\bar{G}_{\alpha}\left(\varepsilon_{0}+z\right)$ at $\bar{E}_{\alpha}$ can then be written as $2 i \bar{J}_{\alpha}\left(\varepsilon_{0}+\bar{E}_{\alpha}\right)$, where the positive energy $\bar{J}_{\alpha}\left(\varepsilon_{0}+\bar{E}_{\alpha}\right)$ gives a measure for the inverse life-time. In fact, as shown by (I. 14.10), the inverse life-time is

$$
\Gamma_{\alpha}=\bar{N}_{\alpha} \bar{J}_{\alpha}\left(\varepsilon_{0}+\bar{E}_{\alpha}\right) \text {. }
$$

Without any loss of generality we can limit our considerations to excited states $|\alpha\rangle$ which are either one-particle states $|k ;\rangle$ (one particle in addition to the Fermi sea) or one-hole states $|; m\rangle$. We have to make an assumption concerning the perturbed excitation energies $\bar{E}_{k}$ and $\bar{E}_{m}$ of such states (we recall that $\vec{E}_{m}$ is defined as negative). We shall namely suppose that the energies $\bar{E}_{k}$ and $\bar{E}_{m}$ have their minimum values for particles and holes at the surface of the Fermi sphere, an assumption which is quite reasonable in our case. Introducing the perturbed Fermi energy $\bar{E}_{F}$, which is equal to $\bar{E}_{k}$ and $-\bar{E}_{m}$ for $|k|=|m|=k_{F}$, our assumption is expressed by

$$
\bar{E}_{k}>\bar{E}_{F} \text { for }|k|>k_{F},
$$

and

$$
\bar{E}_{m}>-\bar{E}_{F} \text { for }|m|<k_{F} \text {. }
$$

To decide whether the states considered are stationary or only metastable, we study the functions $\bar{G}_{k}\left(\varepsilon_{0}+z\right)$ and $\bar{G}_{m}(\varepsilon+z)$ which were defined in I by the formulae

$$
\begin{aligned}
\delta\left(k^{\prime}-k\right) \bar{G}_{k}\left(\varepsilon_{0}+z\right) & =\left\langle k^{\prime}\left|\left[-V+V \bar{D}\left(\varepsilon_{0}+z\right) V-\ldots\right]_{i d C}\right| k\right\rangle, \\
\delta\left(m^{\prime}-m\right) \bar{G}_{m}\left(\varepsilon_{0}+z\right) & =\left\langle m^{\prime}\left|\left[-V+V \bar{D}\left(\varepsilon_{0}+z\right) V-\ldots\right]_{i d C}\right| m\right\rangle .
\end{aligned}
$$

From these equations and the inequality (4.2), it can be shown that the line of discontinuity of the function $\bar{G}_{k}\left(\varepsilon_{0}+z\right)$ is the same for all $k$ and runs from $\bar{E}_{F}$ to $+\infty$, while the line of discontinuity of $\bar{G}_{m}\left(\varepsilon_{0}+z\right)$ always runs from $-\bar{E}_{F}$ to $+\infty$. This has, as one can easily see, the consequence that for arbitrary $|k|>k_{F}$ the function $\bar{G}_{k}\left(\varepsilon_{0}+z\right)$ is not continuous at $\bar{E}_{k}$, whereas $\bar{G}_{m}\left(\varepsilon_{0}+z\right)$ is not continuous at $\bar{E}_{m}$. Thus the states considered are nonstationary.

We limit ourselves to a brief outline of the proof of this important property for the case of $\bar{G}_{k}\left(\varepsilon_{0}+z\right)$. The argument for $\bar{G}_{m}\left(\varepsilon_{0}+z\right)$ is similar. The essential point is that in (4.3) one has among others intermediate states with energies $\bar{E}_{\gamma}$ smaller than $\bar{E}_{k}$ (This situation is in contrast with the case in field theory where, in diagonal diagrams, the energies in the intermediate states are always larger than the energy of the state considered). The proof consists of two steps. In the tirst step one shows that the line of discontinuity of $\bar{G}_{k}\left(\varepsilon_{0}+z\right)$ extends from $\bar{E}_{F}$ to $+\infty$ when $|k|=k_{F}$. The second step extends this conclusion to the case $|k|>k_{F}$. 
It must be emphasized that the validity of the proof rests entirely on the use of the equations (4.3) and (4.4), where the energy shift in the intermediate states is explicitly taken into account. The use of more conventional formulae for $\bar{G}_{k}\left(\varepsilon_{0}+z\right)$ and $\bar{G}_{m}\left(\varepsilon_{0}+z\right)$ involving the unperturbed energy denominators $\left(\varepsilon_{\gamma}-z\right)^{-1}$ would easily lead to erroneous results.

Having thus indicated why no perturbed stationary states correspond to the unperturbed states $|k ;\rangle$ and $|; m\rangle$ of one particle and one hole respectively, we still have to show that, for momenta $k$ and $m$ close to the surface of the Fermi sphere, these states are metastable. A close examination of different approximate expressions for $\bar{J}_{k}\left(\varepsilon_{0}+x\right)$ and $\bar{J}_{m}\left(\varepsilon_{0}+x\right)$, which can be obtained from (4.3) and (4.4), reveals that, for $|k|$ and $|m|$ close to $k_{F}$ and for small $x>0, \bar{J}_{k}\left(\varepsilon_{0}+\bar{E}_{F}+x\right)=\mathrm{O}\left(x^{2}\right), \bar{J}_{m}\left(\varepsilon_{0}-\bar{E}_{F}+x\right)=\mathrm{O}\left(x^{2}\right)$. This behaviour immediately implies that $\bar{J}_{k}\left(\varepsilon_{0}+\bar{E}_{k}\right)$ and $\bar{J}_{m}\left(\varepsilon_{0}+\vec{E}_{m}\right)$ are proportional to $\left(\bar{E}_{k}-\bar{E}_{F}\right)^{2}$ and $\left(\bar{E}_{m}+\bar{E}_{F}\right)^{2}$ respectively, for momenta near the Fermi momentum. These quantities are consequently small, thus ensuring the metastable character of the states considered.

The conclusion that a state of one (additional) particle is not stationary but, for not too high energies, metastable is in agreement with experiments on the scattering of nucleons on heavy nuclei. It is found experimentally that slow nucleons travel a considerable, though finite, distance through nuclear matter before their kinetic energy gets absorbed to form a compound nucleus. The results of such experiments can be analysed by means of the optical model 7), where the interaction between the nucleon and the nucleus is described by a square well potential with a small imaginary component. The imaginary part of this potential corresponds exactly with the decay constant $\Gamma_{k}=\bar{N}_{k} \bar{J}_{k}\left(\varepsilon_{0}+\bar{E}_{k}\right)$ given by (4.1).

On the basis of the Brueckner approximation, Brueckner, Eden, and Francis ${ }^{6}$ ) derived a formula for the imaginary part of the optical potential. This formula can also be obtained from the exact formulae (4.1) and (4.3) if a number of approximations are made. We neglect in (4.3) all terms except the first and replace the function $\bar{D}_{\gamma}\left(\varepsilon_{0}+z\right)$ in the intermediate state $|\gamma\rangle$ by $\left(E_{\gamma}-z\right)^{-1}$. The latter approximation was already discussed in section 2 ; it involves among others the omission of the factor $\bar{N}_{y}$ belonging to the intermediate state. The decay constant $\Gamma_{k}$ is then easily found to be

$$
\delta\left(k^{\prime}-k\right) \Gamma_{k}=\bar{N}_{k} \pi \int \mathrm{d} \gamma\left\langle k^{\prime}|V| \gamma\right\rangle\langle\gamma|V| k ;\rangle \delta\left(\bar{E}_{\gamma}-\bar{E}_{k}\right),
$$

a formula which reduces to the formula of Brueckner, Eden and Francis if also the factor $\bar{N}_{k}$ belonging to the state $|k ;\rangle$ is replaced by one. As stated before, the omission of the two factors $\bar{N}_{k}$ and $\bar{N}_{\gamma}$ can easily lead to considerable errors in the result. Our formula (4.1) provides, however, the basis for more accurate calculations of the inverse life-time of metastable states.

Acknowledgements. The author is indebted to Professor L. V a n $\mathrm{H}$ ove for 
many helpful discussions and for his continued interest. The author also wishes to thank Professor L. Rosenfeld for his hospitality at the theoretical Institute of Manchester and Dr. E. J. Eden for many fruitful discussions.

This work is part of the research program of the "Stichting voor Fundamenteel Onderzoek der Materie", which is financially supported by the Netherlands Organization for pure scientific Research (Z.W.O.).

Received 13-4-57.

\section{REFERENCES}

1) Hugenholtz, N. M., Physica 23 (1957) 481 (this issue).

2) See e.g., Brueckner, K. A. and Levinson, C. A., Phys. Rev, 97 (1955) 1344; also Bethe, H. A., Phys. Rev, 103 (1956) 1353, where an extensive list of references to other work of Brueckner and coll. can be found.

3) Swiatecki, W. J., Phys. Rev. 103 (1956) 265.

4) Bethe, H. A., Phys. Rev. 103 (1956) 1353.

5) Euler, H., Z. Physik 105 (1937) 553.

Huby, R., Proc. Phys. Soc. (London) A 62 (1949) 62.

6) Brueckner, K. A., Eden, R. J. and Francis, N. C., Phys. Rev. 100 (1955) 891.

7) Feshbach, H., Porter, C. E. and Weisskopf, V. F., Phys. Rev. 96 (1954) 448. 